\title{
COMPORTAMENTO ALIMENTAR: RISCO DE COMPULSÃO E RELAÇÃO COM O ESTADO NUTRICIONAL DE ADOLESCENTES
}

\section{FOOD BEHAVIOR: RISK OF COMPULSION AND RELATION WITH THE NUTRITIONAL STATUS OF ADOLESCENTS}

\author{
Naryelle da Rocha Silva ${ }^{1}$ \\ Mielle Neiva Santos ${ }^{2}$ \\ Carla Campos Muniz Medeiros ${ }^{3}$ \\ Fernanda Caroline Tavares de Melo ${ }^{4}$ \\ Danielle Franklin de Carvalho ${ }^{5}$ \\ Ana Raquel de Andrade Barbosa Ribeiro ${ }^{6}$
}

RESUMO: OBJETIVO: verificar o comportamento alimentar dos adolescentes, identificando a ocorrência de compulsão e sua relação com o estado nutricional de escolares. MÉTODO: Estudo transversal realizado em escolas públicas de ensino médio, com 540 adolescentes entre 15 e 19 anos. Foram avaliadas variáveis sociodemográficas, antropométricas e variáveis relacionadas com o estilo de vida, como tabagismo, prática de atividade física, sedentarismo, consumo e comportamento alimentar, sendo o último através de uma versão adaptada do Questionário Holandês de Frequência Alimentar. RESULTADOS: $66,5 \%$ dos adolescentes eram do sexo feminino, com idade média de $16,8( \pm 1,02)$ anos. A média de circunferência abdominal foi de $71,3( \pm 8,7) \mathrm{cm}$ e $21,4( \pm 3,8)$ de Índice de Massa Corporal. Obteve-se 0 escore de compulsão alimentar com três classificações: sem compulsão alimentar $(86,1 \%)$, com compulsão alimentar moderada $(13,3 \%)$ e com compulsão alimentar grave $(0,6 \%)$. Adolescentes com estado nutricional alterado foram os que obtiveram maiores escores de compulsão

\footnotetext{
1 Nutricionista; Docente da Faculdade Maurício de Nassau - CG; Mestre em Saúde Pública; Doutoranda em Saúde da Criança e do Adolescente - UFPE.

${ }^{2}$ Nutricionista; Docente da Faculdade de Ciências Médicas - CG; Mestre em Saúde Pública - UEPB.

${ }^{3}$ Médica Endocrinologista; Docente da Universidade Estadual da Paraíba - UEPB; Doutora em Saúde da Criança e do Adolescente - FCM-UNICAMP.

${ }^{4}$ Enfermeira; Mestranda em Saúde Pública - UEPB.

${ }^{5}$ Farmacêutica-Bioquímica; Docente da Universidade Estadual da Paraíba - UEPB; Doutora em Saúde da Criança e do Adolescente - UFPE.

${ }^{6}$ Médica Endocrinologista; Docente da Universidade Federal de Campina Grande - UFCG; Mestre em Saúde Pública - UEPB.
} 
$(20,2 \%)$. CONCLUSÃO: O estado nutricional mostrou-se relacionado ao escore de compulsão alimentar e à circunferência abdominal.

Palavras chave: Estado Nutricional; Comportamento do Adolescente; Comportamento alimentar.

ABSTRACT: OBJECTIVE: verify the eating behavior of adolescents, identifying the occurrence of compulsion and its relationship with the nutritional status of schoolchildren. METHOD: Cross-sectional study carried out in public high schools, with 540 adolescents between 15 and 19 years old. Sociodemographic, anthropometric variables and variables related to lifestyle, such as smoking, physical activity, sedentary lifestyle, consumption and eating behavior were evaluated, the latter being an adapted version of the Dutch Frequency Questionnaire. RESULTS: $66.5 \%$ of the adolescents were female, with a mean age of 16.8 ( \pm 1.02$)$ years. The mean abdominal circumference was $71.3( \pm 8.7) \mathrm{cm}$ and $21.4( \pm 3.8)$ Body Mass Index. The binge eating score was obtained with three classifications: without binge eating (86.1\%), with moderate binge eating (13.3\%) and with severe binge eating (0.6\%). Adolescents with altered nutritional status were those who had higher compulsion scores (20.2\%). CONCLUSION: Nutritional status was related to binge eating score and abdominal circumference.

Keywords: Nutritional Status; Adolescent Behavior ; Food behavior. 


\section{INTRODUÇÃO}

Hábitos alimentares podem exercer influência sobre o comportamento alimentar e alterar o consumo dos alimentos. Formados desde a infância, tais hábitos perduram durante o crescimento, em família e na sociedade, sendo as preferências e padrões de consumo reflexo dos alimentos que se tornaram familiares (MELO et al., 2017; BARBOSA et al., 2016).

A adolescência é um período marcado por alterações físicas, ambientais, nutricionais e psicológicas onde o indivíduo começa a adquirir independência, inclusive alimentar (PEREIRA, 2015; BARUFALDI, 2016). Influências sofridas nesta etapa da vida, principalmente pelo "poder" da mídia, corroboram para a internalização de padrões corporais que refletem nos comportamentos alimentares, que passam a adquirir, em sua maioria, caráter restritivos (FORTES, 2015).

Para se sentirem bem com seus corpos e alcançar o corpo desejado, alguns adolescentes optam por dietas milagrosas, exercícios físicos extenuantes, ingestão de diuréticos, uso de esteroides anabolizantes, autoindução de vômitos, restrições alimentares excessivas ou jejuns prolongados (TAVARES, 2012; MIRANDA, 2014). Tais desordens alteram a relação dos indivíduos com o alimento, gerando comportamentos restritivos, purgativos e compulsivos, isolados ou articulados (BITTENCOURT, 2013). Em alguns casos, alterações de comportamento alimentar podem futuramente compor transtornos alimentar (TA) (MIRANDA, 2014), como anorexia, com ausência de alimentação; e bulimia, pela ingesta excessiva de alimentos, seguida ou não por vômitos provocados, ambos mais frequentes meninas (MIRANDA, 2014; CAMPOS, 2012).

A busca pelo corpo desejado, magro e "sarado", leva os adolescentes a utilizarem práticas danosas à saúde (MIRANDA, 2014) que podem gerar carências nutricionais, e consequentes condições insatisfatórias de saúde. Existe, entre o estado nutricional "que não incomoda" e os transtornos alimentares, um intervalo onde o estado nutricional é obtido através de métodos compensatórios não 
adequados (MATTOS, 2009). Este intervalo tem sido chamado de Transtorno Alimentar Não Especificado (TANE), caracterizado por transtornos com aspectos clinicamente importantes, mas que não preenchem os critérios de classificação para bulimia e anorexia (GONÇALVES, 2013).

Estudos descritos por autores (GONÇALVES, 2013; ZANELLA, 2012) relatam que indivíduos com estado nutricional adequado tendem a possuir alterações de comportamento alimentar e de autoimagem, como também os não eutróficos, demonstrando relação entre estado nutricional e alterações de comportamento alimentar.

Com base no exposto, este estudo foi desenvolvido com o objetivo de verificar o comportamento alimentar de escolares adolescentes, verificando a ocorrência de compulsão, e sua relação com o estado nutricional. A preocupação é identificar precocemente alterações de comportamento, evitando que hábitos considerados pontuais e, muitas vezes, sem importância, possam se tornar transtornos de difícil tratamento.

\section{METODOLOGIA}

Estudo transversal realizado nas escolas públicas de ensino médio do município de Campina Grande-PB, entre setembro de 2012 e junho de 2013. Para o cálculo amostral, considerou-se uma estimativa de proporção de $50 \%$, erro de $5 \%$, efeito do desenho (deff) de 1,5 e um acréscimo de $3 \%$ para eventuais perdas ou recusas, perfazendo uma amostra final de 570 escolares. Ao final, foi possivel obter um total de 540 questionários totalmente preenchidos, o que representa aproximadamente $95 \%$ da amostra definida estatisticamente. A distribuição deu-se em 39 turmas de 18 escolas, sendo estas de pequeno (até 300 alunos), médio (de 301 a 500) e grande (mais de 500 alunos) porte. As unidades amostrais foram as turmas, sorteadas aleatoriamente, e destas, foram incluídos todos os alunos, desde que atendessem aos critérios de inclusão e aceitassem participar da pesquisa, mediante consentimento escrito, seu ou de seus pais e/ou responsáveis, de acordo 
com a faixa etária. Foram excluídos aqueles que não concluíram os procedimentos necessários à construção do escore PDAY e o preenchimento do questionário de comportamento alimentar, necessário para avaliação do escore de compulsão.

As variáveis estudadas foram: sociodemográficas, como idade, sexo, cor, escolaridade materna; variáveis antropométricas, como peso, altura, circunferência abdominal e estado nutricional (definido pelo índice de massa corporal - IMC); e variáveis relacionadas com o estilo de vida, como tabagismo, prática de atividade física, sedentarismo, comportamento alimentar e compulsão.

Esta última foi avaliada através de uma versão adaptada do Questionário Holandês de Frequência Alimentar (QHCA), validado por Wardle (1987) e traduzido para o português por Almeida, Loureiro e Santos (ALMEIDA et al., 2001). Esse instrumento avalia os estilos alimentares e é composto originalmente por 33 questões, divididas em três subescalas: a) alimentação restrita: estilo alimentar relativo ao conhecimento de hábitos nutricionais adequados; b) alimentação emocional: relativo ao estado emocional do indivíduo; e c) alimentação externa: relativo aos atrativos de aroma e sabor dos alimentos, bem como com a alimentação associada às situações sociais. A pontuação máxima possível nesta escala é de 33 pontos. Considera-se que, quanto maior a pontuação, menor a capacidade de controle alimentar e, quanto menor a pontuação, maior a capacidade de controle: 17 pontos ou menos indicam que não há compulsão alimentar; entre 18 e 26 indica compulsão alimentar moderada e mais de 27 pontos aponta para compulsão alimentar grave (KAKESHITA, 2008).

Além das questões que compõem este questionário, foram adicionadas 19 questões (que não interferem na composição do escore), baseadas na literatura pesquisada (BRASIL, 2010; KANNO, 2008), de forma que a versão final totaliza 52 questões. O propósito é poder avaliar o comportamento tanto através do escore quanto através das práticas individualizadas, avaliadas nas diferentes questões.

Durante a aferição de peso e altura foram seguidos os procedimentos recomendados pela Organização Mundial de Saúde (WHO, 2006). O IMC foi construído a partir da razão do peso (em kilogramas) pelo quadrado da altura (em metros). Além de avaliação como variável contínua, foi utilizado para classificação do estado nutricional, de acordo com o escore z e IMC, segundo a idade: baixo peso 
(3,1 a 1,99), eutrofia ( $\geq 2 \mathrm{a} \leq 1)$, sobrepeso ( $\geq 1 \mathrm{a} \leq 2)$, obesidade. Para os maiores de 18 anos, os pontos de corte do IMC $\left(\mathrm{em} \mathrm{Kg} / \mathrm{m}^{2}\right)$ foram: baixo peso $(<17,5)$, eutrofia $(\geq 17,5 \mathrm{IMC}<25,0)$, sobrepeso $(\geq 25,0 \mathrm{IMC}<30,0)$ e obesidade $(\geq 30,0)$ (CONDE, 2006; BRASIL, 2010).

Para a realização da antropometria, foram seguidos os procedimentos da WHO, 1995. Os dados antropométricos foram coletados em duplicata, sendo considerado o valor médio das duas aferições. Para obtenção do peso foi utilizada balança digital Tanita ${ }^{\circledR}$, com capacidade para $150 \mathrm{~kg}$ e precisão de $0,1 \mathrm{~kg}$. A altura foi aferida através de estadiômetro portátil da marca Tonelli ${ }^{\circledR}$, com precisão de 0,1 $\mathrm{cm}$. Para circunferência abdominal utilizou-se fita métrica inextensível (NCEP, 2002).

Para este estudo, o tabagismo foi classificado considerando-se duas categorias: fumantes (pelo menos 1 cigarro/dia) e não fumante (LEE et al., 2005).

Para a descrição da atividade física acumulada, foram combinados os tempos e as frequências com que foram realizadas atividades como: deslocamento para a escola (a pé ou de bicicleta), aulas de educação física na escola e outras atividades físicas extra-escolares. Foram consideradas as seguintes categorias, agrupadas para fins de análise: 1) inativo e insuficientemente ativo (praticavam atividade física até 299 minutos); e ativo (praticaram 300 minutos ou mais de atividade física).

Para o sedentarismo, foi utilizado o tempo do dia despendido na frente da televisão, computador ou videogame (denominado "tempo de tela"). Foi considerado sedentário o adolescente que ficou duas ou mais horas/dia nestas atividades (BRASIL, 2010).

Os questionários foram duplamente digitados e submetidos à validação no sub- programa Validate do Epi Info 5.3.4, utilizado juntamente com o Statistical Package for the Social Sciences (SPSS, versão 22.0) para o processamento das análises estatísticas.

As medidas de comparação de dois grupos e os valores dos demais fatores de risco e variáveis avaliadas foram feitas aplicando-se o teste de comparação para amostras independentes $\mathrm{t}$ de student e em caso de comparações múltiplas, foi realizada análise de variância, desde que os critérios de normalidade e homogeneidade das variâncias dos grupos fossem atendidos (avaliado pelo Teste 
de Bartlett). Em caso negativo, foram realizados testes não paramétricos equivalentes.

Foi realizada regressão linear simples para verificar a associação do estado nutricional com variáveis sociodemográficas, antropométricas e clínicas; bem como do risco cardiovascular com a compulsão alimentar e fatores de risco clínicos, bioquímicos e antropométricos. Em seguida, foi construído um modelo de regressão linear multivariado para o melhor ajuste das variáveis explicativas. Adotou-se o método hierárquico backward. Para toda a estatística foi adotado o nível de significância de $5 \%$.

O estudo foi aprovado pelo Comitê de Ética em Pesquisa da Universidade Estadual da Paraíba (CAEE: 0077.0.133.000-12). Além disso, foi solicitada à Secretaria Estadual de Educação uma autorização Institucional para coleta de dados nas escolas públicas estaduais de ensino médio.

\section{RESULTADOS}

A amostra foi composta por 540 adolescentes, com média de idade de 16,8 $( \pm 1,0)$, variando entre 15,0 e 19,8 anos. A maioria era do sexo feminino (66,5\%), não branco $(79,1 \%)$, com mais de oito anos de estudo materno $(63,2 \%)$, pertencentes às classes C, D e E $(69,4 \%)$. Destaca-se uma alta prevalência de sedentarismo $(79,1 \%)$ e de inatividade/insuficiência na atividade física $(58,9 \%)$ e uma baixa taxa de tabagismo $(1,9 \%)$ (Tabela 1$)$. 
Tabela 1 - Distribuição dos adolescentes quanto às características socioeconômicas e demográficas e de estilo de vida. Campina Grande-PB. 2012-2013.

\begin{tabular}{|c|c|c|c|c|c|}
\hline \multirow[t]{2}{*}{ Variável $^{a}$} & \multicolumn{2}{|c|}{ Masculino } & \multicolumn{2}{|c|}{ Feminino } & \multirow[t]{2}{*}{$\mathbf{p}$} \\
\hline & $\mathbf{N}$ & $\%$ & $\mathrm{n}$ & $\%$ & \\
\hline Branca & 38 & 34,5 & 72 & 65,5 & 0,81 \\
\hline $\begin{array}{l}\text { Não branca } \\
\text { Escolaridade materna }(n=533)\end{array}$ & 139 & 33,3 & 278 & 66,7 & \\
\hline$<8$ anos & 59 & 30,1 & 137 & 69,9 & 0,17 \\
\hline $\begin{array}{l}\geq 8 \text { anos } \\
\text { Classe econômica }\end{array}$ & 121 & 35,9 & 216 & 64,1 & \\
\hline E a C1 B2 a A1 & $\begin{array}{c}115 \\
66\end{array}$ & $\begin{array}{l}30,7 \\
40,0\end{array}$ & $\begin{array}{c}260 \\
99\end{array}$ & $\begin{array}{l}69,3 \\
60,0\end{array}$ & 0,03 \\
\hline Prática de atividade física & & & & & \\
\hline Inativo/ insuficiente ativo (0 a $299 \mathrm{~min}$ ) & 74 & 23,3 & 244 & 76,7 & 0,00 \\
\hline $\begin{array}{l}\text { Ativo }(\geq 300 \mathrm{~min}) \\
\text { Sedentarismo }\end{array}$ & 107 & 48,2 & 115 & 51,8 & \\
\hline$<2$ horas & 46 & 40,7 & 67 & 59,3 & 0,06 \\
\hline $\begin{array}{l}\geq 2 \text { horas } \\
\text { Tabagismo }(n=539)\end{array}$ & 135 & 31,6 & 292 & 68,4 & \\
\hline Não fumante & 177 & 33,5 & 352 & 66,5 & \\
\hline Fumante & 4 & 40 & 6 & 60 & $0,73^{b}$ \\
\hline
\end{tabular}

A Houve variação no total de respostas referente aos estudantes que rejeitaram fornecer a informação; b Teste exato de Fisher.

Os adolescentes apresentaram média de 71,3 $( \pm 8,7) \mathrm{cm}$ de circunferência abdominal e 21,4 $( \pm 3,8)$ de Índice de Massa Corporal. Pela classificação do estado nutricional, teve-se: 23 casos de baixo peso (4,3\%), 423 eutróficos (78,3\%), $69 \mathrm{com}$ sobrepeso $(12,8 \%)$ e 25 obesos $(4,6 \%)$. Para fins de análise, o estado nutricional foi reagrupado: baixo peso e eutrófico formaram uma categoria, e sobrepeso e obesidade a outra. Nesse caso, o grupo composto por indivíduos de baixo peso e eutróficos foi $82,6 \%$ ( $n=446)$ da amostra, enquanto os com sobrepeso e obesidade foram $17,4 \%(n=94)$.

O questionário de comportamento alimentar original era subdividido em três blocos: alimentação restrita, alimentação emocional e alimentação externa. A distribuição dos adolescentes que forneceram respostas afirmativas segundo as 
variáveis de comportamento alimentar está descrita na Tabela 2, de acordo com seu estado nutricional.

Tabela 2 - Distribuição dos adolescentes que responderam "sim" às questões de comportamento alimentar, com diferenças estatisticamente significantes entre as categorias do estado nutricional. Campina Grande-PB. 2012-2013.

\begin{tabular}{|c|c|c|c|c|c|}
\hline Variáveis de comportamento alimentar & & Estac & utric & & \\
\hline & & & & ado & \\
\hline riáveis relativas à "Alimentação Restrita" & $\mathbf{n}$ & $\%$ & $\mathbf{n}$ & $\%$ & $r$ \\
\hline $\begin{array}{l}\text { cê tenta comer menos do que gostaria nos } \\
\text { rários das refeições? }\end{array}$ & 100 & 22,4 & 53 & 56,4 & $<0,01$ \\
\hline $\begin{array}{l}\text { ocê recusa comida ou bebida oferecidos a } \\
\text { ocê por estar preocupado(a) com seu peso? }\end{array}$ & 54 & 12,1 & 46 & 48,9 & $<0,01$ \\
\hline $\begin{array}{l}\text { uando você come muito em um dia, você } \\
\text { me menos que o habitual nos dias seguintes? }\end{array}$ & 92 & 20,6 & 53 & 56,4 & $<0,01$ \\
\hline $\begin{array}{l}\text { ocê come menos com o objetivo de ficar mais } \\
\text { lagro? }\end{array}$ & 58 & 13,0 & 61 & 64,9 & $<0,01$ \\
\hline $\begin{array}{l}\text { ocê já ficou longos períodos de tempo sem } \\
\text { mer para ficar mais magro(a)? }\end{array}$ & 45 & 10,2 & 30 & 32,6 & $<0,01$ \\
\hline $\begin{array}{l}\text { ocê já comeu bem mais que o normal e depois } \\
\text { ovocou vômito para ficar mais magro(a)? }\end{array}$ & 11 & 2,5 & 10 & 10,8 & $<0,01$ \\
\hline $\begin{array}{l}\text { ocê tenta não comer entre as refeições por que } \\
\text { stá observando seu peso? }\end{array}$ & 33 & 7,4 & 37 & 39,4 & $<0,01$ \\
\hline $\begin{array}{l}\text { ocê tenta não comer à noite por que está } \\
\text { reocupado com seu peso? }\end{array}$ & 28 & 6,3 & 39 & 41,5 & $<0,01$ \\
\hline $\begin{array}{l}\text { ocê faz as três refeições principais (café da } \\
\text { lanhã, almoço e jantar) diariamente? }\end{array}$ & 312 & 70,7 & 55 & 58,5 & 0,02 \\
\hline cê "pula" alguma refeição? & 165 & 37,3 & 49 & 52,1 & 0,01 \\
\hline lariável relativa à “Alimentação Emocional” & $\mathbf{n}$ & $\%$ & $\mathbf{n}$ & $\%$ & $\boldsymbol{P}$ \\
\hline $\begin{array}{l}\text { ocê tem vontade de comer quando não tem } \\
\text { ada para fazer? }\end{array}$ & 336 & 75,3 & 61 & 64,9 & 0,05 \\
\hline lariáveis relativas à "Alimentação Externa" & $\mathbf{n}$ & $\%$ & $\mathbf{n}$ & $\%$ & $\boldsymbol{P}$ \\
\hline $\begin{array}{l}\text { a comida é saborosa, você come mais que o } \\
\text { abitual? }\end{array}$ & 399 & 89,5 & 73 & 77,7 & $<0,01$ \\
\hline $\begin{array}{l}\text { e você tem algo delicioso ao seu alcance para } \\
\text { omer, você come imediatamente? }\end{array}$ & 308 & 69,1 & 46 & 48,9 & $<0,01$ \\
\hline lariável relativa à "Alimentação Cotidiana" & $\mathbf{n}$ & $\%$ & $\mathbf{n}$ & $\%$ & $P$ \\
\hline eus pais têm uma aliment & 362 & 81,3 & 59 & 62,8 & $<0,01$ \\
\hline
\end{tabular}

Com base nas questões do QHCA obteve-se o escore de compulsão alimentar, que foi inicialmente dividido em três classificações: sem compulsão 
alimentar ( $n=465, \%=86,1)$, com compulsão alimentar moderada $(n=72, \%=13,3)$ e com compulsão alimentar grave $(n=3, \%=0,6)$. Posteriormente o escore foi recategorizado em indivíduos com compulsão e sem compulsão. Os indivíduos classificados com compulsão compuseram 13,9\% $(n=75)$ da amostra. Dos adolescentes com compulsão alimentar 20,2\% ( $n=19)$ apresentavam-se com estado nutricional alterado, enquanto apenas $12,5 \%(n=56)$ dos adolescentes com estado nutricional normal possuíam algum nível de compulsão.

A Tabela 3 apresenta a análise estatística de regressão linear simples, relacionando o Índice de Massa Corporal com as variáveis compulsão alimentar, idade, atividade física, sedentarismo e circunferência abdominal.

Tabela 3 - Regressão linear simples do Índice de Massa Corporal com as variáveis compulsão alimentar, idade, atividade física, sedentarismo e circunferência abdominal. Campina Grande-PB. 2012-2013.

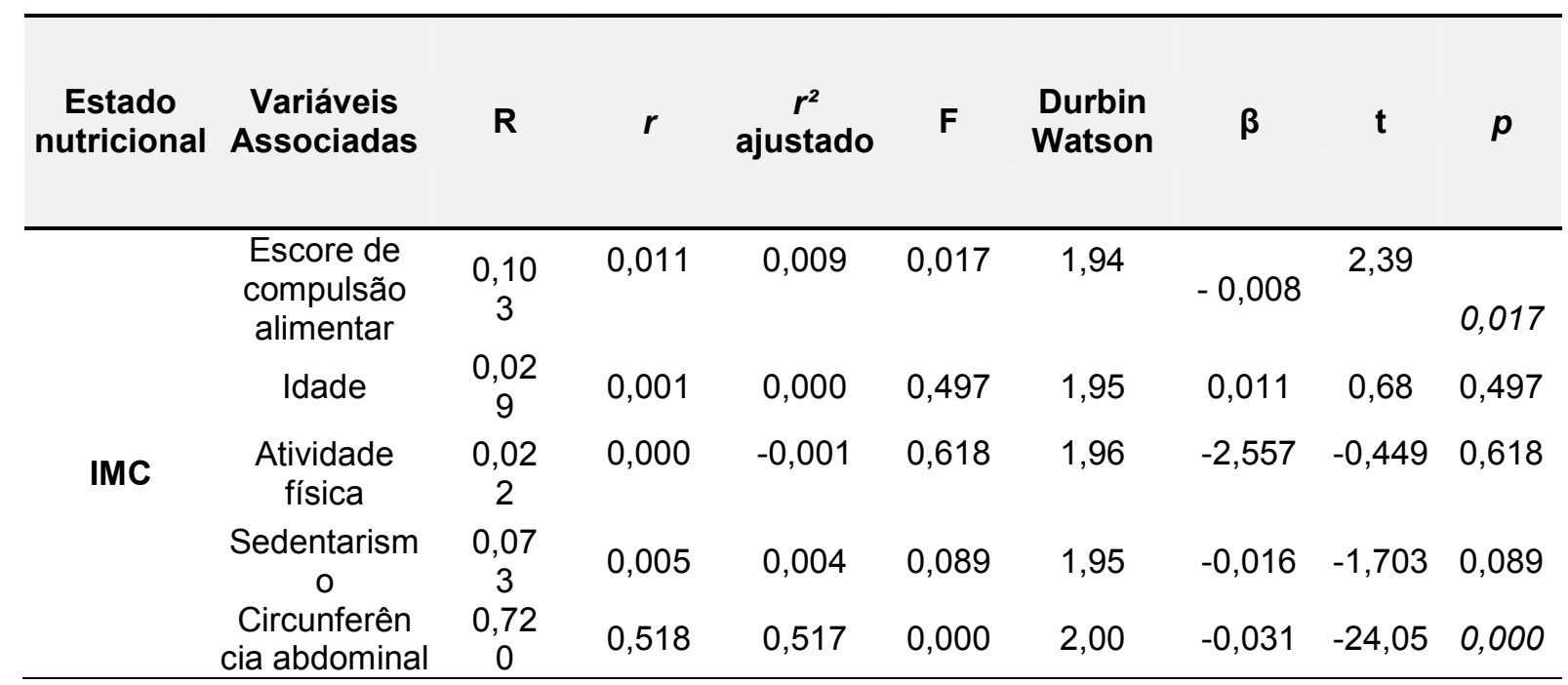

De acordo com o resultado do coeficiente de determinação ajustado $\left(r^{2}\right)$ para a relação do IMC com a circunferência abdominal, esta variável é a que mais interfere no IMC (51,7\%), seguida pela compulsão alimentar $(0,9 \%)$. O teste de Durbin Watson apresentou relação positiva em todas as variáveis que, com valores próximos de 2, explicam o bom ajuste da regressão linear. 


\section{DISCUSSÃO}

Os participantes possuíam predominância das classes econômicas C, D e E $(69,4 \%)$, embora a escolaridade materna fosse igual ou maior a 8 anos $(63,2 \%)$, o que pode influenciar de forma positiva a alimentação da família (BARUFALDI, 2016). O baixo índice de tabagismo (1,9\%), entretanto, difere do descrito por Lacerda e Cardoso (2009), que obtiveram relação direta entre o alto nível de escolaridade dos pais e a maior probabilidade do fumo pelos adolescentes.

O sedentarismo elevado $(79,1 \%)$ e os altos percentuais de inatividade física ou atividade física insuficiente $(58,9 \%)$ não reverteram em alteração de estado nutricional. Com sedentarismo aliado a baixos níveis de atividade física, o esperado era que os adolescentes estivessem acima do peso, mas a maioria dos adolescentes possuía estado nutricional normal (82,6\%). Sendo a circunferência abdominal a variável que mais interferiu no IMC $(51,7 \%)$, o que sugere uma relação positiva entre a elevação de ambos.

O QHCA aponta, além do escore de compulsão, algumas variáveis que podem ser analisadas individualmente. Nos questionamentos acerca do comportamento alimentar merecem destaque algumas perguntas, que estavam divididas em blocos, descritos a seguir.

Nas variáveis relativas à Alimentação Restrita, o ENA (Estado Nutricional Alterado) apresentou os maiores percentuais de respostas "sim" referentes a hábitos compensatórios diante da alimentação. Comer menos com o objetivo de ficar mais magro, pular refeições e não comer a noite porque está preocupado com o peso são mecanismos de compensação para manutenção do peso corporal, que devem ser observados com atenção. Recomenda-se que a manutenção de peso seja realizada através de modificações no estilo de vida, sem a utilização de métodos compensatórios. Destaca-se que os adolescentes com ENN (Estado Nutricional Normal) relataram fazer as três principais refeições diariamente, característica positiva importante na manutenção do estado nutricional. 
Chama atenção que $10,8 \%$ dos adolescentes com ENA já chegaram a comer bem mais que o normal e depois provocaram vômito para ficar mais magros (as). Provocar vômito com o objetivo de ficar mais magro é um comportamento preocupante, principalmente em se tratando de adolescentes, que estão em franco crescimento e desenvolvimento (BITTENCOURT, 2013).

Esta alteração comportamental pode ser resultante do impacto do conceito propagado pela mídia sobre a magreza e beleza. Adolescentes comparam-se com o padrão imposto e se tornam mais susceptíveis ao uso de diversos métodos possíveis para alcançar o corpo desejado (BITTENCOURT, 2013; LIRA, 2017).

No bloco relativo à Alimentação Emocional, o questionamento sobre a vontade de comer quando não tem nada para fazer obteve alto percentual, tanto no ENN (75,3\%), como no ENA (64,9\%). Ressalta-se que este é, de fato, um comportamento cotidiano comum a população em geral, que se utiliza de lanches e petiscos quando estão ociosos. Preocupa o fato de que estes lanches são frequentemente calóricos e pouco nutritivos (BRASIL, 2010).

Nas variáveis relativas à Alimentação Externa: "Se a comida é saborosa, você come mais que o habitual?", obteve prevalência $89,5 \%$ para os adolescentes com ENN e $77,7 \%$ para os com ENA, demonstrando outro comportamento habitual, de comer mais do que se come normalmente quando o alimento foge à alimentação cotidiana ou mesmo quando chama a atenção pelo sabor e aparência. Na pergunta: "Se você tem algo delicioso ao seu alcance para comer, você come imediatamente? ", que aponta a tendência de não se abster de comer algo que esteja ao seu alcance, 69,1\% dos adolescentes com ENN e 48,9\% dos com ENA responderam afirmativamente. Chama atenção que os adolescentes com ENA se abstêm mais de comer alimentos que os interessem, provavelmente como mecanismo de controle de peso. A resposta cerebral ao impulso de visualização é difícil de ser contida, então comer torna-se um comportamento automático, pois o cérebro gasta mais energia cognitiva para abstenção do que para alimentação por impulso (KAKESHITA, 2008). Nesse sentido, quem tem o ENA mostrou-se mais propenso a evitar alimentação em excesso ou mesmo comer alimentos atrativos imediatamente.

No bloco Alimentação Cotidiana os adolescentes com ENN responderam que consideram que seus pais têm uma alimentação saudável, o que pode ter sido 
referência para sua alimentação e manutenção do estado nutricional eutrófico (BARUFALDI, 2016).

A regressão linear simples apontou associação entre o escore de compulsão alimentar e o estado nutricional, bem como deste com a circunferência abdominal, sendo esta a que explica melhor o modelo, tanto que permaneceu na regressão multivariada.

A compulsão alimentar é um TANE, que pode apresentar-se na forma clínica ou subclínica. Em estudo realizado com adolescentes, a prevalência de casos clínicos de compulsão alimentar foi de 1,6\% e subclínicos, 2,5\%, totalizando $4,1 \%$ de adolescentes com algum nível de compulsão (GONÇALVES, 2013). Em outro estudo, com estudantes de ensino médio e universitárias, a frequência de comportamento alimentar desordenado foi de $6,6 \% .^{8}$ No presente estudo, os adolescentes com compulsão alimentar grave foram $0,6 \%$ e os com compulsão alimentar moderada 13,3\%, totalizando $13,9 \%$ de adolescentes considerados com compulsão alimentar. Chama atenção que a compulsão moderada se destaca, o que pode sugerir a utilização de métodos compensatórios cotidianos que passam despercebidos.

Neste estudo, o IMC mostrou-se associado ao escore de compulsão alimentar, assim como o encontrado por Bittencourt (2013), que ainda apresenta o aumento do IMC como fator de proteção para TA, por estar inversamente relacionado ao risco de desenvolvimento de comportamentos alimentares alterados, evidenciando que a manutenção do estado nutricional pode estar ocorrendo através de métodos compensatórios de controle de ingestão alimentar. Estes métodos, uma vez realizados de forma cotidiana podem levar a transtornos alimentares e comportamentos compulsivos diante da alimentação (GONÇALVES, 2013).

Adolescentes com ENA foram os que obtiveram maiores escores de compulsão (20,2\%). Preocupa saber que adolescentes, que estão em fase de crescimento e demanda energética aumentada, estão apresentando algum nível de compulsão alimentar, pois hábitos formados nessa fase da vida se tornam mais consistentes na vida adulta, podendo agravar-se. Quando se considera que estes adolescentes possuem ENA, há que se observar que o sedentarismo e a atividade 
física insuficiente podem se tornar agravantes na idade adulta, com tendência de aumento no peso ao longo dos anos (ENES, 2010).

Nesse sentido, tem que se orientar a prática alimentar saudável associada à atividade física orientada. O estímulo a uma imagem corporal satisfatória deve estar associado a uma vida mais saudável, procurando o melhor estado nutricional possível, considerando o biótipo e a história familiar.

\section{CONCLUSÃO}

Os dados do presente estudo indicam que os adolescentes podem estar mantendo o estado nutricional através de métodos compensatórios não satisfatórios. Além de terem apresentado um significativo escore de compulsão alterado, características individuais como jejuns prolongados, omissão de refeições e sentimentos de culpa podem se configurar como TANE. A identificação precoce de alterações do comportamento alimentar é importante, no sentido de planejar políticas públicas de modificação do estilo de vida e combate a mecanismos compensatórios de controle do peso.

\section{REFERÊNCIAS BIBLIOGRÁFICAS}

ALMEIDA, G. A. N.; LOUREIRO, S. R.; SANTOS, J. E. Obesidade mórbida em mulheres Estilos alimentares e qualidade de vida. ALAN, v. 51, n. 4, p. 359-65, 2001.

BARBOSA, M. I. C.; OLIVEIRA, B. R.; CARVALHO, N. A, MARTINS KA. Educação alimentar e nutricional: influência no comportamento alimentar e no estado nutricional de estudantes. Mundo Saúde (São Paulo), v. 40, n. 4, p. 399-409, 2016.

BARUFALDI, L. A.; ABREU, G. A. A.; OLIVEIRA, J. S.; SANTOS, D. F.; FUJIMORI, E.; VASCONCELOS, S. M. L. ERICA: prevalência de comportamentos alimentares saudáveis em adolescentes brasileiros. Rev Saúde Pública, v. 50, n. 1, p. 1-6, 2016.

BITTENCOURT, L. J.; NUNES, M. O.; OLIVEIRA, J. J. F.; CARON, J. Risco para transtornos alimentares em escolares de Salvador, Bahia, e a dimensão raça/cor. Rev Nutr (Campinas), v. 26, n. 5, p. 497-508, 2013.

BRASIL. Ministério da Saúde. Secretaria de Vigilância em Saúde. Secretaria de Atenção à Saúde. Política Nacional de Promoção da Saúde. Brasília: Ministério da Saúde; 2010. 60p. 
(Série B. Textos Básicos de Saúde).

CAMPOS, J. G. S. C.; HAACK, A. Anorexia e bulimia: aspectos clínicos e drogas habitualmente usadas no seu tratamento medicamentoso. Comun Ciênc Saúde, v. 23, n. 3, p. 253-262, 2012.

CONDE, W. L.; MONTEIRO, C. A. Body mass index cutoff points for evaluation of nutritional status in Brazilian children and adolescents. J Pediatr, v. 82, n. 4, p. 266-72, 2006.

ENES, C. C.; SLATER, B. Obesidade na adolescência e seus principais fatores determinantes. Rev Bras Epidemiol, v. 13, n. 1, p. 163-71, 2010.

FORTES, L. S.; MEDEIROS, J. F. F.; PAES, S. T.; DIAS, F. C.; CIPRIANI, F. M.; FERREIRA, M. E. C. Associação da internalização dos padrões corporais, sintomas depressivos e comportamento alimentar restritivo em jovens do sexo masculino. Cien Saúde Coletiva, v. 20, n. 11, p. 3457-3465, 2015.

GONÇALVES, J. A.; MOREIRA, E. A. M.; TRINDADE, E. B. S. M.; FIATES, G. R. M. Transtornos alimentares na infância e na adolescência. Rev Paul Pediatr, v. 31, n. 1, p. 96103, 2013.

KAKESHITA, I. S.; ALMEIDA, S. S. Estudo da relação entre o índice de massa corporal e o comportamento alimentar de adultos. Nutire: Rev Soc Bras Alim Nutr, v. 33, n. 1, p. 21-30, 2008.

KANNO, P.; RABELO, M.; MELO, G. Discrepâncias na imagem corporal e na dieta de obesos. Rev Nutr PUCCAMP, v. 21, n. 4, p. 423-30, 2008.

LACERDA, A. C.; CARDOSO, M. F. Smoking among Portuguese teenagers: assessing school, peer and family factors of vulnerability and protection. Revi Port Saúde Pública, v. 27, n. 2, p. 17-26, 2009.

LEE, W. Y.; JUNG, C. H.; PARK, J. S.; RHEE, E. J.; KIM, S.W. Effects of smoking, alcohol, exercise, education, and family history on the metabolic syndrome as defined by the ATP III. Diabetes Res Clin Pract, v. 67, n. 1, p. 70-7, 2005.

LIRA, A. G.; GANEN, A. P.; LODI, A. S.; ALVARENGA, M. S. Uso de redes sociais, influência da mídia e insatisfação com a imagem corporal de adolescentes brasileiras. J Bras Psiquiatr, v. 66, n. 3, p. 164-71, 2017.

MATTOS, R. S.; LUZ, M. T. Sobrevivendo ao estigma da gordura: um estudo socioantropológico sobre obesidade. Physis (Rio J.), v. 19, n. 2, p. 489-507, 2009.

MELO, K. M.; CRUZ, A. C. P.; BRITO, M. F. S. F.; PINHO, L. Influência do comportamento dos pais durante a refeição e no excesso de peso na infância. Esc Anna Nery,

v. 21 , n. 4, p. 1-6, 2017.

MIRANDA, V. P. N.; CONTI, M. A.; BASTOS, R. R.; LAUS, M. F.; ALMEIDA, S. S.; FERREIRA, M. E. C. Imagem corporal de adolescentes de cidades rurais. Cien Saúde Colet, v. 19, n. 6, p. 1791-1801, 2014.

PEREIRA, C. M.; SILVA, A. L.; SÁ, M. I. Fatores que influenciam os comportamentos alimentares: questionário das escolhas alimentares dos adolescentes. Psic saúde e doenças (Lisboa), v. 16, n. 3, p. 421-438, 2015.

SANTOS, V. E. P.; CRUZ, N. M.; MOURA, J. G.; MATOS, K. K. C. O estado nutricional e comportamento alimentar de um grupo de mulheres na estratégia de saúde da família. Rev Enferm UFSM, v. 2, n. 2, p. 394-400, 2012. 
Sociedade Brasileira de Cardiologia (BR). VI Diretrizes Brasileiras de Hipertensão Arterial. Arq Bras Cardiol, v. 95, n, I, p. 1-51, 2010.

TAVARES, A. F.; CAMPANA, A. N. N. B.; MORAES, M. S. Apreciação corporal e modificação da aparência física em estudantes adolescentes de baixo poder aquisitivo. Psicol Esc Educ, v. 16, n. 2, p. 275-282, 2012.

The Third Report of the National Cholesterol Education Program (NCEP). Expert panel on detection. Evaluation, and treatment of high blood cholesterol in adults (Adult Treatment Panel III). JAMA. v. 285, n. 19, p. 2486-97, 2002.

WHO, WORLD HEALTH ORGANIZATION. Child growth standards: Length/height-for- age, weight-for-age, weight-for-length, weight-for height and body mass index-for-age. Methods and development. Geneva (SWZ); 2006.

ZANELLA, A.; SCHMIDT, K. H. Estado nutricional e comportamento alimentar de profissionais de academia de Frederico Westphalen-RS. RBNE (São Paulo), v. 6, n. 35, p. 367-375, 2012. 